\title{
Effets d'un stress hydrique intervenu pendant le stade végétatif et la phase de floraison chez le gombo
}

\author{
Rasmata NANA $^{1^{*}}$, Zoumbiessé TAMINI ${ }^{2}$ et Mahamadou SAWADOGO ${ }^{3}$ \\ ${ }^{1}$ Laboratoire d'Ecophysiologie Végétale, Université de Ouagadougou, 03 BP 848 Ouagadougou 03, \\ Burkina Faso. \\ ${ }^{2}$ Laboratoire d'Ecophysiologie Végétale, Université de Ouagadougou, 03 BP 848 Ouagadougou 03, \\ Burkina Faso. \\ ${ }^{3}$ Laboratoire de Génétique et de biotechnologie des Plantes, Université de Ouagadougou, 03 BP 848 \\ Ouagadougou 03, Burkina Faso. \\ *Auteur correspondant, E-mail : nanaras2001@yahoo.fr
}

\section{RESUME}

Cette étude a pour but d'évaluer l'impact du stress hydrique durant le stade végétatif et à la floraison sur la teneur en proline et en chlorophylle de cinq variétés de gombo. Les variations entre les différentes variétés étudiées ont été détectées. Pour cela, des plantes de cinq variétés de gombo sont cultivées sous trois traitements hydriques : une alimentation hydrique continue (T0), une restriction hydrique au stade végétatif (T1) et une restriction hydrique à la floraison (T2); durant ces traitements, les teneurs en proline et en chlorophylle ont été mesurées. Le stress hydrique a provoqué une augmentation de la teneur en proline foliaire chez toutes les variétés aussi bien au stade végétatif qu'à la floraison. Par ailleurs, sous l'effet du déficit hydrique aux 2 stades de développement, les cinq variétés réagissent par une augmentation du contenu foliaire en chlorophylle totale. Cependant, le rapport chlorophylle a/chlorophylle b diminue sous l'effet du stress hydrique intervenu à la floraison chez toutes les variétés. Vis-à-vis du stress hydrique au stade végétatif, les variétés UAE 19 et UAE 45 ont un rapport chloropyhlle a/chlorophylle b qui augmente, traduisant ainsi une meilleure tolérance contrairement aux variétés UAE 1, UAE 22 et UAE 40.

(C) 2009 International Formulae Group. All rights reserved.

Mots clés : variétés, traitements hydriques, proline, chlorophylle

\section{INTRODUCTION}

Depuis la fin des années 1960, les régions du Sahel subissent une sécheresse (Institut de Recherche pour le Développement, 1996), due à une importante baisse de la pluviométrie et à une augmentation de sa variabilité spatiotemporelle. Elle constitue une menace sérieuse pour l'agriculture.

Jadis considéré comme une culture marginale (Sawadogo et al., 2009), le gombo constitue de nos jours un légume très rémunérateur pour les communautés pauvres du fait de son fort potentiel de vente sur les marchés ruraux et urbains. Il procure des revenus susceptibles de satisfaire de nombreux besoins tant individuels, familiaux que collectifs. Le gombo frais, sec découpé et transformée en poudre, sont acheminés vers le marché extérieur, l'Europe et la sous-région principalement (Agence Américaine pour Développement International, 2006). En outre, le gombo occupe la deuxième place de production légumière après la tomate (Hamon et Charrier, 1997).

Malgré cela, sa culture est souvent confrontée à des régimes pluviométriques déficitaires qui se traduisent par des contraintes hydriques sévères et erratiques. 
Ces conditions hydriques déficitaires limitent l'expression du potentiel de production des variétés et rendent incertain l'espoir des agriculteurs. En effet, au Burkina Faso, la production nationale en fruits du gombo estimée à 1600 tonnes en 1989 a baissé au cours des dernières campagnes agricoles: 1285,159 tonnes en 1995/1996 dont 1116,254 tonnes sont vendues soit $86,86 \%$ de la production; 728 tonnes en 1996/1997; 726 tonnes en $1997 / 1998$; 990 tonnes en $1998 / 1999$ et 1356 tonnes en 2004/2005 (MAHRH / DGPSA / Direction des Statistiques Agricoles, 2006).

Or, le Burkina Faso à l'instar des pays sahéliens fait face à un défi de taille qui est la lutte contre la pauvreté. L'un des aspects prioritaires de cette lutte est la sécurité alimentaire afin de nourrir une population sans cesse croissante. L'assurance de la sécurité alimentaire ne peut être efficace que si des perspectives sont ouvertes pour diversifier les cultures et augmenter la production en dépit des caprices de la pluviométrie. L'adaptation des plantes à la sécheresse, une des préoccupations de la biotechnologie est une perspective efficace et prometteuse pour obtenir des plantes mieux adaptées. De nombreux travaux relatifs à l'adaptation des plantes cultivées à la sécheresse ont été réalisés (Boyer, 1969 ; Adjahossou, 1991; Moulineau, 1993 ; Albouchi et al., 2003 ; Mekliche et al., 2003 ; Bezzala, 2005; Hamidou, 2006). Toutefois l'accumulation de la proline chez les plantes constitue un véritable mécanisme de tolérance aux contraintes hydriques, (Moulineau, 1993 ; Ibriz et al., 1993, Tahri et al., 1998 ; Slama et al., 2004). Par ailleurs, le rapport chlorophylle a/chlorophylle b est considéré comme un bon indicateur du seuil de tolérance au déficit hydrique (Hireche, 2006). Ces paramètres biochimiques et physiologiques d'adaptation à la sécheresse aussi performants soit-ils, ne sont étudiés et utilisés que dans la sélection des céréales (Hamidou, 2006). Très peu de travaux à caractères physiologiques ont été menés sur les légumes notamment sur le gombo.

Afin d'assurer une bonne production du gombo, la recherche des variétés tolérantes et/ou résistantes au déficit hydrique devient une nécessité. Pour ce faire, l'étude des paramètres physiologiques et biochimiques de résistance à la sécheresse pourrait contribuer à la réussite d'une sélection variétale.

Le présent travail a pour objectif d'apprécier le comportement de cinq variétés de gombo vis-à-vis des restrictions hydriques et d'évaluer l'impact de cette restriction sur la teneur en proline et en chlorophylle aux stades végétatif et floraison de ces variétés.

\section{MATERIEL ET METHODES \\ Matériel végétal}

Cinq variétés de gombo (UAE 1 ; UAE 19 ; UAE 22 ; UAE 40 ; UAE 45) obtenues par une sélection variétale ayant connue la participation des paysans dans cinq provinces du Burkina Faso ont fait l'objet de notre étude.

Ces variétés ont été choisies pour l'étude parce que d'une part, elles n'ont pas fait l'objet de travaux antérieurs du point de vue de leurs comportements physiologiques vis-à-vis d'un déficit hydrique et d'autre part, ces variétés ont déjà fait l'objet d'une amélioration pour leurs rendements en fruits.

\section{Méthodes de culture}

Les plantes ont été cultivées dans des pots en plastique à raison d'un plant par pot. Ces pots sont de forme conique de diamètre inférieur $27 \mathrm{~cm}$; diamètre supérieur $33 \mathrm{~cm}$; de hauteur $35 \mathrm{~cm}$; de capacité 17 litres et dont le fond est troué pour éviter l'asphyxie racinaire.

Le substrat de culture est un sol sablolimoneux $(63,94 \%$ de sable, $25,93 \%$ de limon et $10,13 \%$ d'argile). Chaque pot contient 17 $\mathrm{kg}$ de terre homogénéisée. Cinq graines sont semées dans chaque pot à une profondeur de 5 $\mathrm{cm}$. Un démariage à 1 plant par pot a été effectué le $14^{\text {ème }}$ jour après semis (JAS).

\section{Dispositif expérimental}

L'essai compte au total 90 pots (5 variétés x 2 pots/variété $\times 3$ variantes du traitement $\mathrm{x} 3$ répétitions) disposés en «splitplot ». Les traitements représentent les sous blocs et les pots correspondent aux parcelles élémentaires. Les blocs constituent les répétitions. 
R. NANA et al. / Int. J. Biol. Chem. Sci. 3(5): 1161-1170, 2009

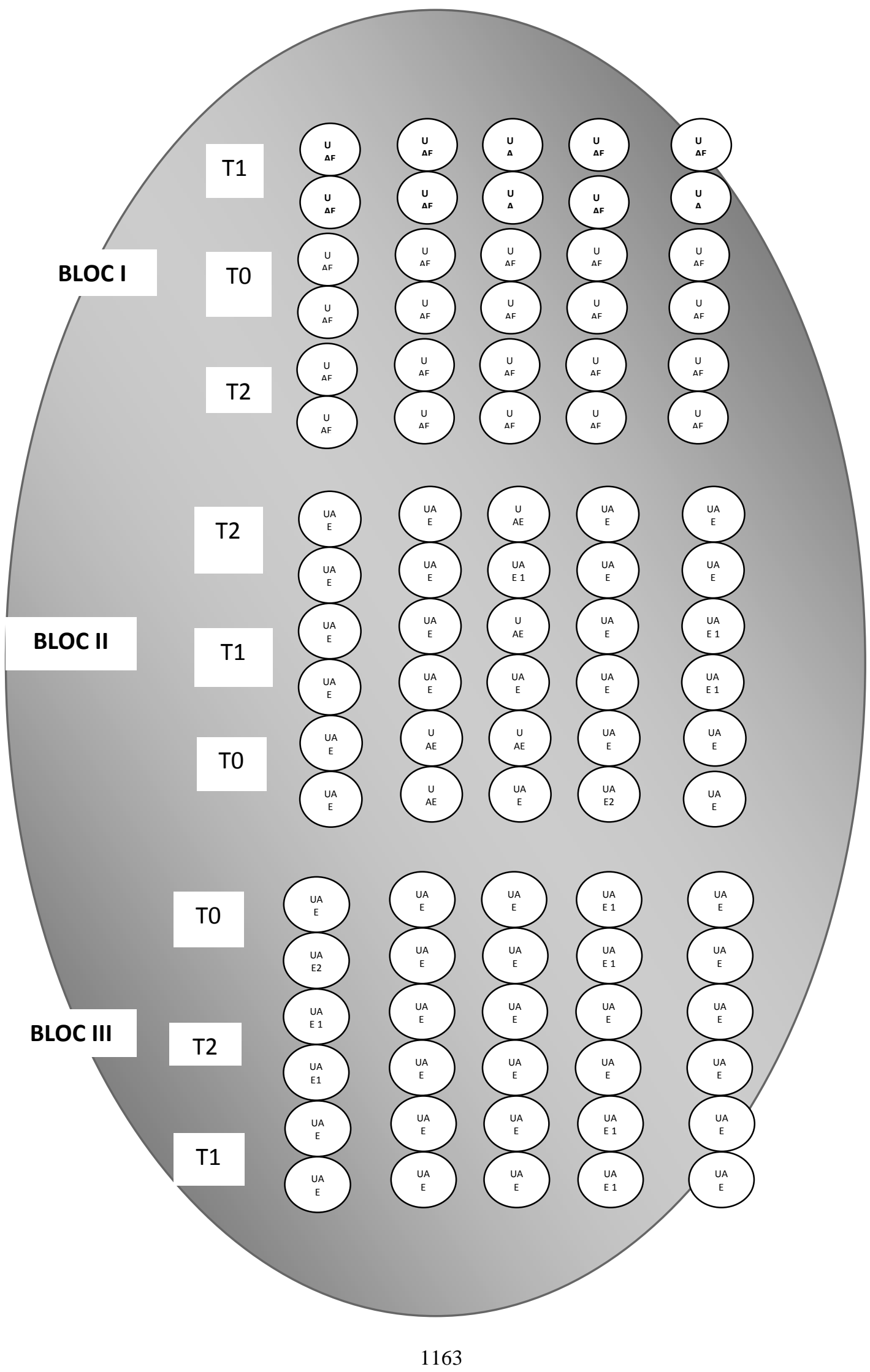




\section{Traitement hydrique}

Trois niveaux de régime hydrique ont été appliqué: Traitement témoin (T0) avec une alimentation hydrique non limitant, arrosé chaque 2 jours; Traitement avec restriction hydrique au stade végétatif (T1) et un Traitement avec restriction hydrique à la floraison (T2). Le traitement $\mathrm{T} 1$ a débuté le $32^{\text {ème }}$ Jours après semis (JAS) et a duré 13 jours ; le traitement $\mathrm{T} 2$ est intervenu à partir du $52^{\text {ème }}$ jusqu'au $61^{\text {ème }}$ JAS soit 9 jours de stress hydrique. Avant l'application du stress hydrique tous les plants étaient soumis au même traitement, soit un arrosage chaque 2 jours.

Le prélèvement des échantillons de feuille à été effectué le dernier jour de stress sur la $3^{\text {ème }}$ feuille à partir de l'apex et stocké dans de l'azote liquide à $195{ }^{\circ} \mathrm{C}$ pour les mesures.

\section{Méthodes de mesures \\ Teneur en proline des feuilles}

La teneur en proline est déterminée par la méthode de Ough adaptée par Bogdanov (1999). Les solutions utilisées sont de l'acide formique $100 \%$, une solution de ninhydrine à $3 \%$ dans de l'éthylène glycol, une solution aqueuse de proline à $0,032 \mathrm{mg} / \mathrm{ml}$, une solution aqueuse de 2-propanol $50 \%$ et les solutions aqueuses d'extrait de feuilles à $0,025 \mathrm{~g} / \mathrm{ml}$.

Le principe consiste à mettre $0,5 \mathrm{ml}$ d'extrait de feuille dans un tube, $0,5 \mathrm{ml}$ d'eau distillée dans un second tube (essai blanc) dans un second tube et $0,5 \mathrm{ml}$ de solution standard de proline dans trois autres tubes.

Dans chaque tube, on ajoute $1 \mathrm{ml}$ d'acide formique et $1 \mathrm{ml}$ de solution de ninhydrine. Ces tubes sont soigneusement couverts et agités pendant $15 \mathrm{mn}$ à température ambiante avec un INCUBATOR SHAKER. Les tubes sont ensuite placés dans un bain marie bouillante pendant $15 \mathrm{mn}$. A ces tubes, on a ajouté 2,5 $\mathrm{ml}$ de 2-propanol $50 \%$ et replacé dans un bain marie $70{ }^{\circ} \mathrm{C}$ pendant $10 \mathrm{mn}$. Les tubes sont alors refroidis et les absorbances des mélanges sont lues au spectrophotomètre à $510 \mathrm{~nm}$. La teneur en proline est déterminée selon la formule suivante :

Proline $(\mathrm{mg} / \mathrm{g}$ de feuille fraîche $)=$ [Es/Ea x E1/E2 x80]/1000
Es = absorbance de la solution d'échantillon ; $\mathrm{Ea}=$ absorbance de la solution standard de proline (moyenne de 3 échantillons) ; E1 = mg de proline pris pour la solution standard; $\mathrm{E} 2=\mathrm{g}$ de feuille fraîche séchée ; 80 = facteur de dilution.

\section{Teneur en chlorophylle (Chl)}

La teneur en chlorophylle est déterminée par la méthode de Mckinney (1941). Elle consiste à broyer $100 \mathrm{mg}$ de matière fraîche en présence d'acétone $80 \%$. Après filtration, la densité optique est lue à 663 et $645 \mathrm{~nm}$. Les concentrations en chlorophylles a et $\mathrm{b}$ sont déduites par les formules suivantes :

$$
\begin{aligned}
& \text { chl } a=12\left(\text { Do 663) }-2,67\left(D_{0} 645\right)\right. \\
& \text { chl } b=22,5\left(D_{0} 645\right)-4,68\left(D_{0} 663\right)
\end{aligned}
$$

$$
\text { DO = Densité Optique }
$$

La teneur en chlorophylle totale est déduite de la somme des chlorophylles a et b.

\section{Traitement et analyse statistique des données}

Les données sont calculées sous forme d'une moyenne de 3 répétitions à l'aide du logiciel EXCEL 2007 et les graphiques également réalisés à l'aide du même logiciel.

Les résultats obtenus ont fait l'objet d'analyse de la variance des moyennes pour évaluer la signification de l'effet seuil $\mathrm{P}<0,05$ par rapport à la plus petite différence significative avec le test de Fischer. Les tests de comparaison des moyennes sont effectués avec le test REGWQ pour sa fiabilité. Toutes ces analyses ont été réalisées avec le logiciel XLSTAT version 7.1.

\section{RESULTATS \\ Teneur en proline libre}

Les figures $1 \mathrm{a}$ et $1 \mathrm{~b}$ montrent l'impact d'un déficit hydrique survenu au stade végétatif et à la floraison sur la teneur en proline chez les cinq variétés de gombo. Comparativement aux plantes témoins arrosées chaque deux jours, celles soumises à une restriction hydrique au stade végétatif, et floraison connaissent une augmentation de la teneur en proline foliaire (Figure 1). Cette accumulation est plus importante chez la variété UAE 40 que chez les quatre autres variétés aussi bien au stade végétatif qu'à la 
floraison, respectivement 6,48 et $8,55 \mathrm{mg} / \mathrm{g}$ de matière fraîche.

Les valeurs de la probabilité $\mathrm{P}$ relative à l'analyse de variance montrent qu'il n'existe pas de différences significatives entre les variétés au stade végétatif $(\mathrm{P}=0,41)$ et également à la phase floraison $(\mathrm{P}=0,22)$. Pourtant les données laissent apparaître que les variétés UAE 40 et UEA 45 ont les plus fortes teneurs en proline respectivement sous l'effet du stress hydrique à la floraison et au stade végétatif (Figure 2).

Par ailleurs, le tableau 1 qui établit les paramètres de modèle de l'ANOVA sur la teneur en proline montre que la variété UAE 1 qui exprime des valeurs nulles d'intervalle de confiance est la variété de référence. Les autres variétés UEA 19 , UAE 22, UAE 40 et UAE 45 présentent zéro dans leur intervalle de confiance caractérisée par les bornes inférieures et supérieur 95\%. Ces variétés ne diffèrent pas de la variété UAE 1, donc le facteur variété n'a pas d'influence sur la teneur en proline.

\section{Teneur en chlorophylle totale (chlorophylle $\mathbf{a}+\mathbf{b}$ )}

Parallèlement à l'augmentation de la teneur en proline foliaire, on observe également une augmentation de la teneur en chlorophylle totale (chlorophylle $a+b$ ) chez toutes les variétés durant les stress hydriques survenu aux deux stades de développement. L'analyse de la variance appliquée aux résultats montre un coefficient de détermination $\left(\mathrm{R}^{2}\right)$ de 0,621 pour le stress hydrique à la phase végétative et 0,483 avec la restriction hydrique à la floraison (Tableau 2). Ceci indique que $62,1 \%$ de la variabilité au stade végétatif peut être expliquée par le traitement et seulement $48,3 \%$ de la variation à la floraison est expliquée par le traitement. Au regard de la table ANOVA (Tableau 3), aucune différence significative n'apparaît entre les variétés $(\operatorname{Pr}=0,12)$. Cependant, la probabilité associée à $\mathrm{F}$ pour le facteur traitement est $<0,0001$, ceci implique un risque $<0,01 \%$ qui est suffisamment inférieur au risque d'erreur choisi qui est de $5 \%$. Donc le facteur traitement a une influence très significative sur la teneur en chlorophylle totale. En effet, le test REGWQ (Tableau 4) indique que deux paires de traitement sont significativement différentes (T0 T1 et T0 T2).

Contrairement à l'augmentation de la teneur en chlorophylle totale, le rapport chlorophylle a/chlorophylle b (chla/chlb) subi une baisse chez les cinq variétés sous le stress hydrique à la floraison et seulement chez les variétés UAE 1, UAE 22 et UAE 40 sous la restriction hydrique au stade végétatif (Figure 3). D'un point de vue statistique, il n'y a pas de différence significative pour le facteur variétal (Tableau 5). Par contre, les trois modalités du traitement hydrique appliqué ont un effet significatif sur le rapport chla/chlb.

\section{DISCUSSION}

Le dosage de la teneur en proline foliaire réalisé chez les cinq variétés révèle que les plantes stressées réagissent par une augmentation de la teneur en proline quel que soit leur stade de développement. Cette accumulation de la proline permet aux plantes de supporter le manque d'eau par une diminution du potentiel osmotique. $\mathrm{Ce}$ phénomène est connu sous le terme d'ajustement osmotique. De toutes les variétés, la UAE 40 se montre plus accumulatrice, elle est suivie de la UAE 45 ; ce qui traduit un meilleur ajustement osmotique de ces deux variétés par rapport aux autres variétés. Bezzalla (2005) rapporte que l'accumulation de la proline résulte de la perturbation du métabolisme des protéines causée par une protéolyse membranaire. Le processus d'accumulation de la proline dans les tissus foliaires est considéré comme un critère d'adaptation aux stress par plusieurs auteurs (N'da , 1984 ; Monneveux et Nemmar, 1986; Moulineau, 1993; Tahri et al., 1998 ; Mekliche et al., 2003; Bezzala, 2005; Hamidou, 2006 ; Hireche, 2006). L'augmentation de la teneur en chlorophylle totale chez toutes les variétés sous la restriction hydrique au stade végétatif et à la floraison révèle que le stress hydrique provoqué par une baisse de la quantité d'eau disponible entraîne une concentration du pigment photosynthétique chez le gombo. Atti et al. (2004) ont soulignés une diminution du contenu foliaire en chlorophylle chez le soja lorsque les plants subissent un stress hydrique 
sévère. Le stress hydrique appliqué serait modéré pour les plants de gombo.

L'augmentation de la chlorophylle comme celle de la proline foliaires sous l'effet $\mathrm{du}$ stress hydrique suggère que ces deux composés ont un précurseur commun, le glutamate (Reddy et Veeranjaneyulu, 1991). Il existerait donc des voies distinctes de biosynthèse de ces composés. Il semble que la stimulation de la synthèse de la proline soit parallèle à une activation globale d'une voie métabolique partant du glutamate semi- aldéhyde et conduisant à la proline (Levigneron et al., 2005). Un signal enzymatique au niveau du chloroplaste qui déclencherait la biosynthèse de la chlorophylle, stimulerait également celle de la proline dans cet organite.

Dans les conditions de stress hydrique à la floraison (Figure 4b), toutes les variétés réagissent par une diminution du rapport chl $\mathrm{a} / \mathrm{chl} \mathrm{b}$. Ces résultats suggèrent que l'appareil photosynthétique des plants de gombo

Tableau 1: Paramètres du modèle.

\begin{tabular}{lcccccc}
\hline \multicolumn{1}{c}{ Paramètre } & Valeur & $\begin{array}{c}\text { Ecart- } \\
\text { type }\end{array}$ & $\begin{array}{c}\text { t de } \\
\text { Student }\end{array}$ & Pr > t & $\begin{array}{c}\text { Borne } \\
\text { inférieure } \\
\mathbf{9 5} \%\end{array}$ & $\begin{array}{c}\text { Borne } \\
\text { supérieure } \\
\mathbf{9 5} \%\end{array}$ \\
\hline Constante & 4,031 & 1,039 & 3,879 & 0,001 & 1,909 & 6,153 \\
Variété-1 & 0,000 & - & - & - & - & - \\
Variété-19 & $-0,088$ & 1,469 & $-0,060$ & 0,952 & $-3,089$ & 2,913 \\
Variété-22 & $-0,050$ & 1,469 & $-0,034$ & 0,973 & $-3,051$ & 2,951 \\
Variété-40 & 0,251 & 1,469 & 0,171 & 0,865 & $-2,750$ & 3,252 \\
Variété-45 & $-0,778$ & 1,469 & $-0,529$ & 0,600 & $-3,779$ & 2,223 \\
\hline \multicolumn{1}{r}{$=$ variable de Student; $\mathrm{Pr}=$ probabilité } & & & &
\end{tabular}

Tableau 2 : Coefficients d'ajustement pour le stress hydrique aux stades végétatif et de floraison.

\begin{tabular}{lcc}
\hline & Stade végétatif & Stade de floraison \\
\hline $\mathbf{R}$ & 0,788 & 0,695 \\
$\mathbf{R}^{2}$ & 0,621 & 0,483 \\
$\mathbf{R}^{2}$ ajusté & 0,542 & 0,375 \\
\hline
\end{tabular}

$\mathrm{R}=$ coefficient de corrélation, $\mathrm{R}^{2}$ = coefficient de détermination, $\mathrm{R}^{2}$ ajusté. = coefficient de détermination ajusté.

Tableau 3 : Table ANOVA.

\begin{tabular}{cccccc}
\hline Source de variation & ddl & $\begin{array}{c}\text { Somme des } \\
\text { carrés }\end{array}$ & $\begin{array}{c}\text { Carré } \\
\text { moyen }\end{array}$ & F de Fisher & Pr $>$ F \\
\hline Variétés & 4 & 508,966 & 127,241 & 1,940 & $0,124^{\mathrm{ns}}$ \\
Traitements & 2 & 2596,881 & 1298,440 & 19,801 & $<0,0001^{* * *}$ \\
\hline
\end{tabular}

*** = différence hautement significative $; \mathrm{ns}=$ différence non significative $;$ ddl $:$ degré de liberté $; \mathrm{F}=$ variable de Fischer $;$ $\operatorname{Pr}=$ probabilité. 
Tableau 4: Analyse des différences entre les groupes pour la modalité traitement au seuil de 5\%.

\begin{tabular}{ccccccc}
\hline Modalités & Différence & $\begin{array}{c}\text { Différence } \\
\text { réduite }\end{array}$ & $\begin{array}{c}\text { Valeur } \\
\text { critique }\end{array}$ & $\begin{array}{c}\text { Probabilité }> \\
\text { Différence }\end{array}$ & $\begin{array}{c}\text { Alpha } \\
\text { (modifié) }\end{array}$ & Significatif \\
\hline $\mathrm{T} 0 \sim \mathrm{T} 1$ & 16.125 & 4.569 & 2.429 & 0.000 & 0.050 & $* * *$ \\
$\mathrm{~T} 1 \sim \mathrm{T} 2$ & 0.516 & 0.146 & 2.018 & 0.885 & 0.050 & $\mathrm{~ns}$ \\
$\mathrm{~T} 2 \sim \mathrm{T} 0$ & 15.609 & 4.423 & 2.018 & $<0.0001$ & 0.050 & $* * *$ \\
\hline
\end{tabular}

*** = différence très significative $; \mathrm{ns}$ : différence non significative; $\mathrm{T} 0=$ traitement témoin $; \mathrm{T} 1=$ traitement avec arrêt d'arrosage au stade végétatif ; $\mathrm{T} 2$ = traitement avec arrêt d'arrosage à la floraison.

Tableau 5 : Analyse de variance du rapport chlorophylle a/chlorophylle b, signification de $\mathrm{F}$ et groupe des moyennes.

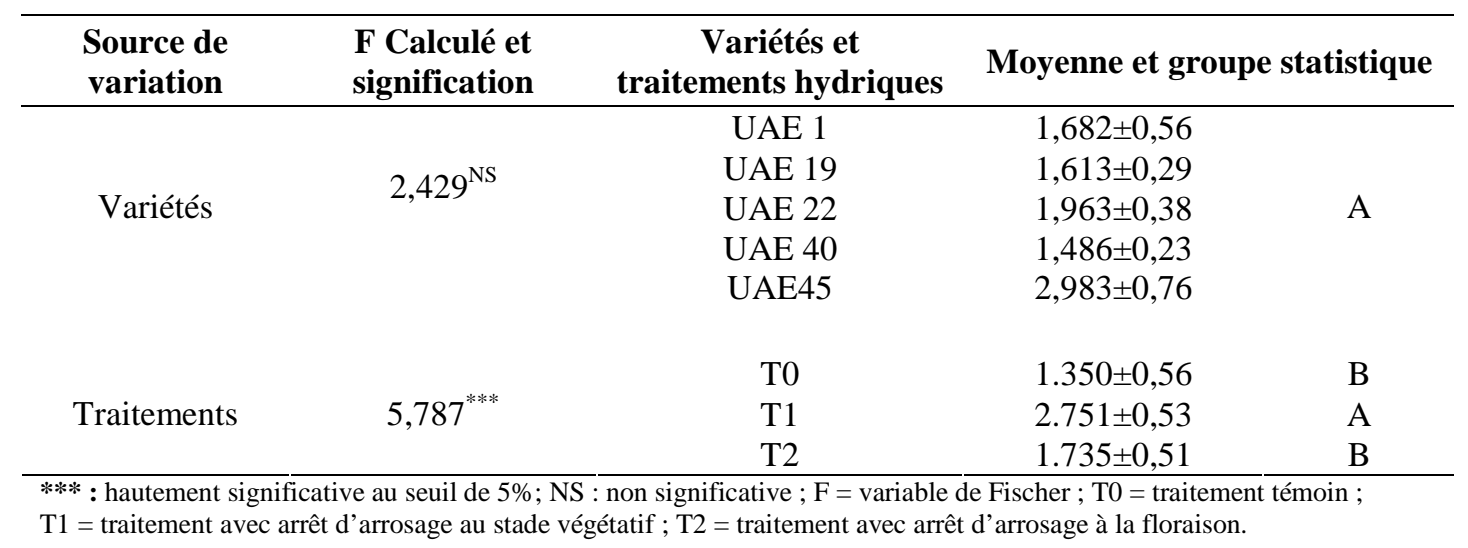

घT0 घT2
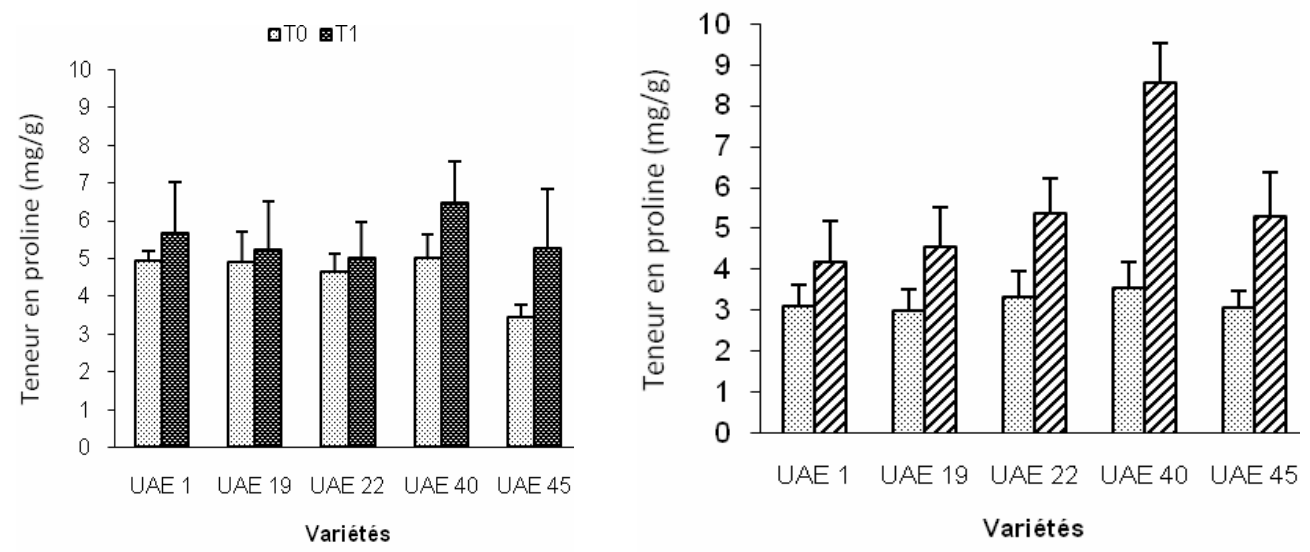

Figure 1: Evolution de la teneur en proline foliaire de cinq variétés de gombo sous un déficit hydrique au stade végétatif et à la floraison. 

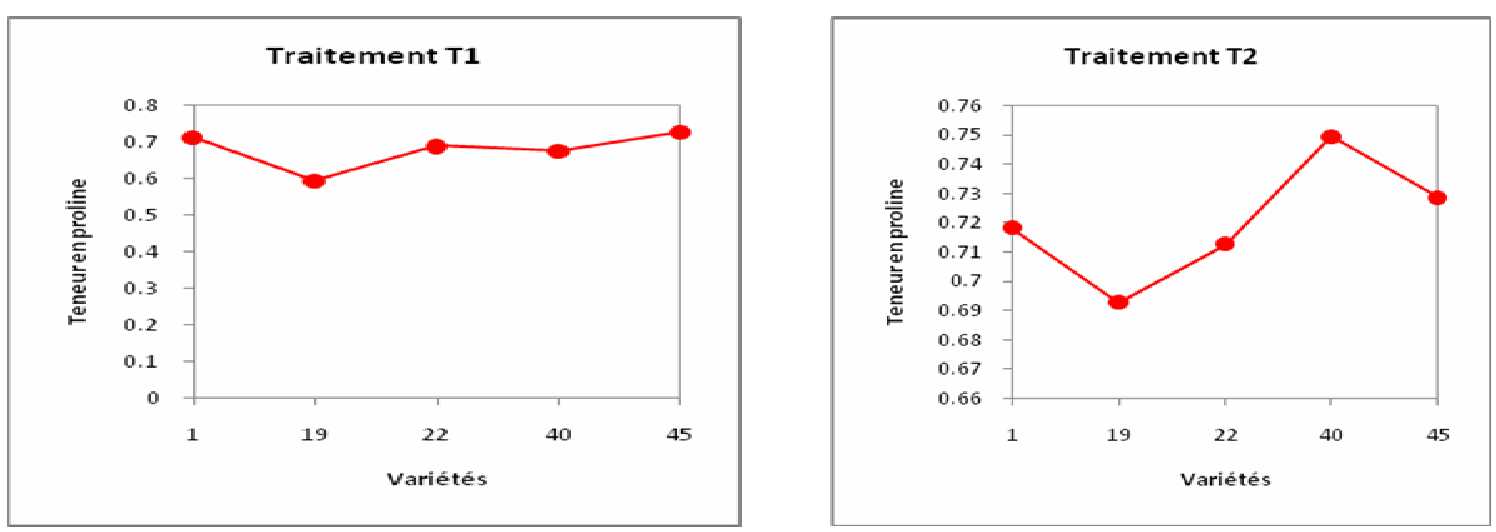

Figure 2 : Graphique des moyennes.
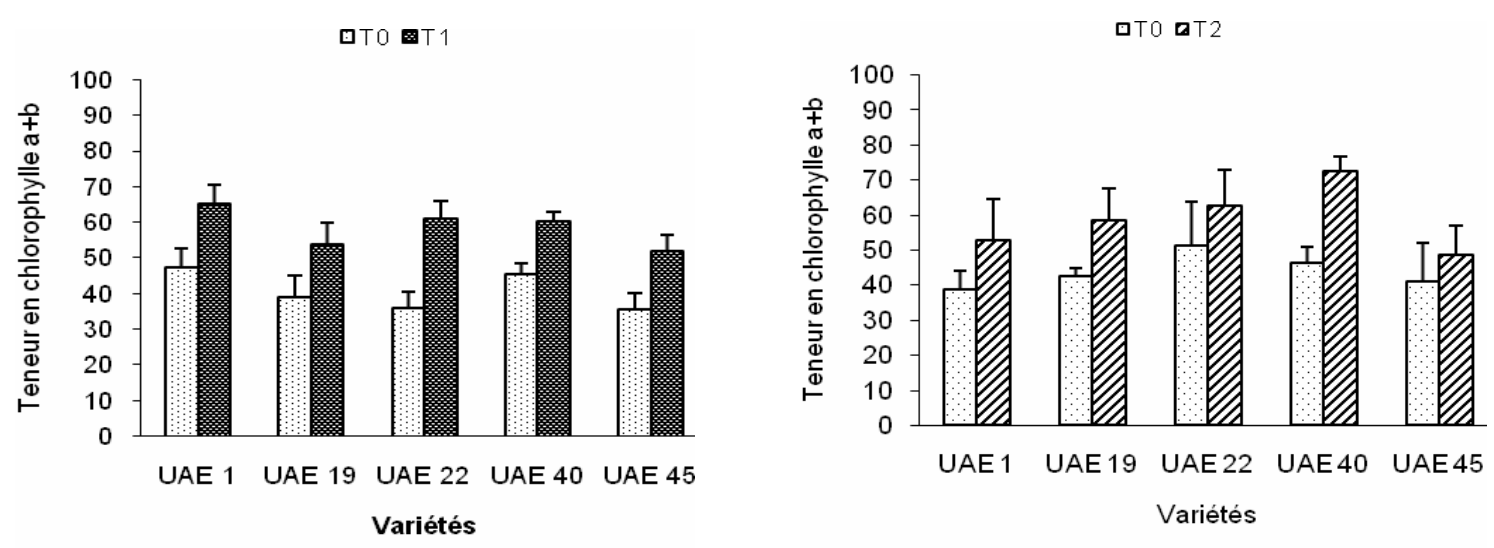

Figure 3a

Figure $3 \mathbf{b}$

Figure 3 : Variation de la teneur en chlorophylle totale chez les cinq variétés en fonction des traitements hydriques.
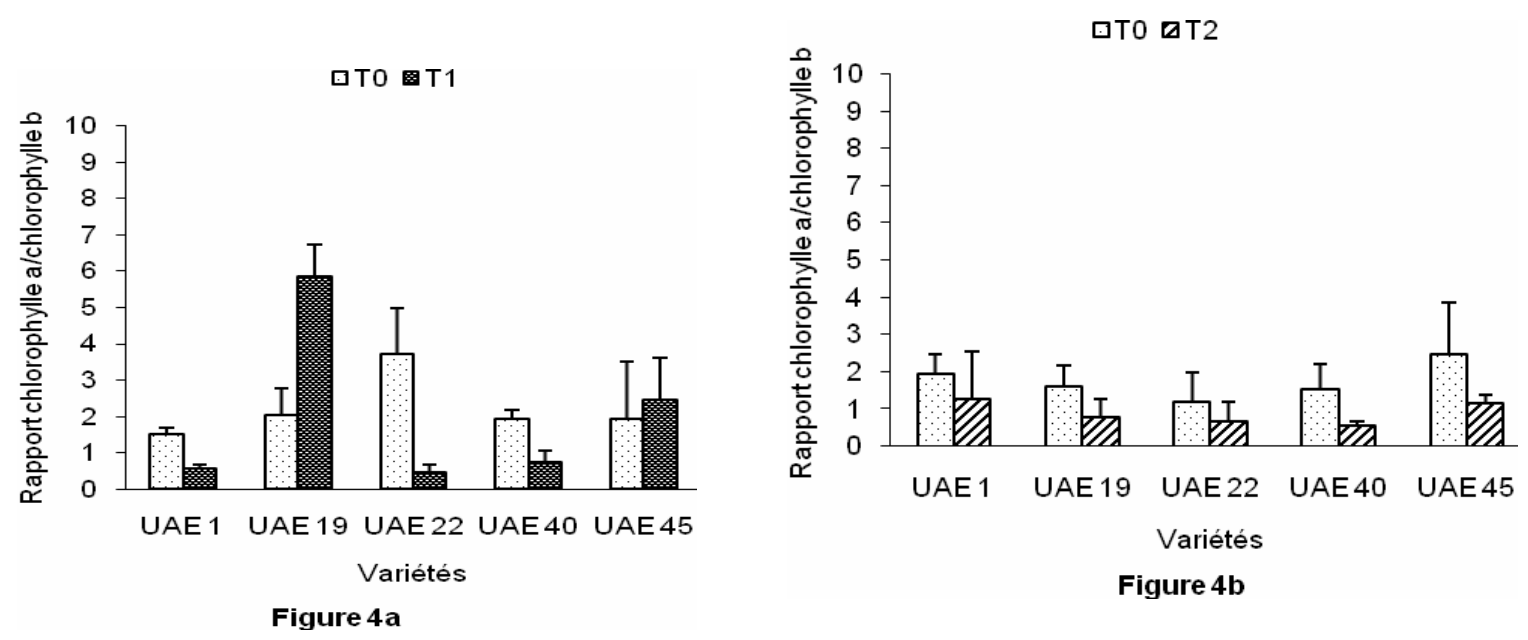

Figure 4 : Variation du rapport chlorophylle a / chlorophylle b des cinq variétés en fonction du traitement hydrique. 
présente une adaptation au manque d'eau au stade floraison par une diminution du rapport chlorophylle a/chlorophylle b.

Cependant, au stade végétatif le comportement des cinq variétés est dissemblable. En effet, le déficit hydrique a entraîné chez les variétés UAE 19 et UAE 45 une augmentation du rapport chl a/ chl b. Selon Guettouche (1990), plus ce paramètre est élevé, plus la variété est tolérante au manque d'eau. Sur cette base, nous pouvons donc dire que seules les variétés UAE 19 et UAE 45 tolèrent le stress hydrique au stade végétatif. Par contre, toutes les variétés se montrent vulnérables au stress hydrique à la floraison. On note donc une tolérance variétale au stade végétatif.

\section{Conclusion}

L'adaptation au déficit hydrique s'est traduite par une augmentation de l'accumulation de la proline et de la teneur en chlorophylle totale chez toutes les variétés de gombo. Cependant, il apparaît une différence variétale pour le rapport chl a/chl b pour le stress hydrique appliqué au stade végétatif indiquant une meilleure tolérance des variétés UAE 19 et UAE 45 à ce stade de développement. Toutefois, les cinq variétés se sont montrées moins tolérantes au manque d'eau à la floraison. L'accumulation de la proline et le rapport chlorophylle $a /$ chlorophylle b lors d'un déficit hydrique peuvent être considérés comme des index de sécheresse.

\section{REFERENCES BIBLIOGRAPHIQUES}

Adjahossou DF. 1991. Les glucides de réserve et la tolérance à la sécheresse chez le palmier à huile. Physiol. des arbres et arbustes en zones arides et semi-arides; groupe d'étude de l'arbre, paris, France ; 117-129.

Agence Américaine pour le Développement International. 2006. Activité de renforcement de commercialisation agricole en Guinée : la filière gombo (okra) en Guinée. PCE-I-00-99-0000300, Task Order No. 29, 32p.

Albouchi A, Bejaoui Z, El aouni MH. 2003. Influence d'un stress hydrique modéré ou sévère sur la croissance de jeunes plants de Cassuarina glauca Sieb. Séch., 14(3) : 137-142.

Atti S, Bonnell R, Smith D, Prasher S. 2004. Response of an indeterminate soybean (Glycine max (L.) Merr) to chronic water deficit during reproductive development under greenhouse conditions. Can. Wat. Ress. J., 29(4): 209-222.

Bezzalla A. 2005. Essai d'introduction de l'arganier (argania spinosa (L.) Skeels) dans la zone M'doukel et évaluation de quelques paramètres de résistance à la sécheresse. Mémoire de Magistère en Sciences agronomique, Université Al Hadj Lakhadar- Batna, Batna, p.143.

Bogdanov S. 1999. Harmonised Methods of the International Honey Commission. Swiss Bee Research Center, FAM, Liebefeld, CH-3003 Bern,Switzerland, p. 62.

Boyer J. 1969. Étude expérimentale des effets $\mathrm{du}$ régime d'humidité du sol sur la croissance végétative, la floraison et la fructification des caféiers robusta. Café Cacao Thé, XIII (3) : 187-200.

Guettouche R. 1990. Contribution à l'identification des caractères morphophysiologiques d'adaptation à la sécheresse chez le blé dur (Triticum durum Desf). Thèse agro. app. , p. 85.

Hamidou F. 2006. Paramètres physiologiques, biochimiques et agronomiques pertinents pour les programmes d'amélioration de l'adaptation du niébé (Vigna unguiculata (L.) Walp.) au déficit hydrique. Thèse, Université de Ouagadougou, Ouagadougou, p.137.

Hamon S, Charrier A. 1997. Les gombos. In Amélioration des Plantes Tropicales (1 $1^{\text {èr }}$ édn). CIRAD; 313-333.

Hireche Y. 2006. Réponse de la luzerne (Medicago stiva L.) au stress hydrique et à la profondeur de semis. Mémoire de Magistère en sciences agronomique, Université Al Hadj Lakhadar-Batna, Batna, p.82.

Ibriz M, Monneveux P, Chery J, Comeau A. 1993. Étude Des Interactions Entre Le Déficit Hydrique et la Jaunisse 
Nanisante chez l'Orge. Ed. AAUPELFUREF. John Libbey Eurotext: Paris ; 185-206.

Institut de Recherche pour le développement. 1996. Afrique tropicale : la sécheresse gagne du terrain. Fiche scientifique $\mathrm{n}^{\circ} 28$.

Levigneron A, Lopez F, Vansuyt G, Berthomieu P, Fourcroy P, CasseDelbart F. 2005. Les plantes face au stress salin. Cah. Agr., 4: 263-73.

MAHRH / DGPSA / Direction des Statistiques Agricoles. 2006. Campagne agricole 2005-2006.

Mckinney G. 1941. Absoption of light by chlorophyll solutions. J. Biol. Chem., 140: 315-322.

Mekliche A, Boukecha D, Hanifi-Mekliche L. 2003. Etude de la tolérance à la sécheresse de quelques variétés de blé dur (Triticum durum Desf.) : effet de l'irrigation de complément sur les caractères phénologiques, morphologiques et physiologiques. Ann. Agron., 24(1 et 2): 97-110.

Monneveux P, Nemmar N. 1986. Contribution à l'étude de la résistance à la sécheresse chez le blé tendre (T. aestivum L.) et le blé dur ( $T$. durum Desf): étude de l'accumulation de la proline au cours du cycle de développement. Agron., 6(6) : 583-590.
Moulineau C. 1993. Variations sous contrainte hydrique de la teneur en acides aminés libres foliaires du mil. 233-44.

N'da KAR. 1984. Réponse physiologique du riz (Oryza sativa L.) au déficit hydrique : étude comparée de deux types culturaux (variété pluviale, variété aquatique) au déficit hydrique. Thèse de doctorat d'ingénieur, Sci. agron., Université de Montpellier, p.148.

Reddy PS, Veeranjaneyulu K. 1991. Proline metabolism in senescing leaves of horsgram (Macrotyloma uniflorum Lam.). J. Plant. Physiol., 137: 381-383.

Sawadogo M, Balma D, Nana R, Sumda RMT. 2009. Diversité agromorphologique et commercialisation du gombo (Abelmoschus esculentus L.) à Ouagadougou et ses environs. Int. J. Biol. Chem. Sci., 3(2): 326-336.

Slama A, Ben S, Zid M. 2004. La proline estelle un osmorégulateur chez le blé dur ? Communication aux $15^{\mathrm{es}}$ Journées biologiques, For. Sci. Biol., pp. 18-21.

Tahri EH, Belabed A, Sadki K. 1998. Effet d'un stress osmotique sur l'accumulation de proline, de chlorophylle et des ARNm codant pour la glutamine synthétase chez trois variétés de blé dur (Triticum durum). Bull. Inst. Sci. 21: 81-87. 